\section{Avaliação dos fatores de risco cardiovasculares em uma população rural brasileira}

\author{
Cardiovascular risk factor assessment in a rural \\ Brazilian population
}

\section{Evaluación de los factores de riesgo cardiovascular en una población rural de Brasil}

\begin{abstract}
This cross-sectional study assessed cardiovascular health in 863 adults in rural communities in the Jequitinhonha Valley, Minas Gerais State, Brazil, focusing on seven factors, four of which behavioral (diet, physical activity, smoking, and body weight) and three biological (total cholesterol, fasting blood glucose, and arterial pressure), classified as ideal, intermediate, or poor Men showed better results in behavioral factors $(p=0.01)$, with more men displaying 2 or 3 factors at ideal levels (48.5 and $25.8 \%$, respectively), when compared to women (44.2 and 19.8\%, respectively) $(p=0.01)$. Women presented more biological factors at ideal levels (19.1\%) when compared to men (7.9\%) $(p<0.001)$. Only three participants $(0.4 \%)$ showed all seven factors at ideal levels. Prevalence of ideal indices was very low, showing the need for strategies to improve cardiovascular health in this population.
\end{abstract}

Cardiovascular Diseases; Rural Population; Risk Factors; Health Promotion

\author{
Mariana Santos Felisbino-Mendes 1 \\ Ann Kristine Jansen 1 \\ Crizian Saar Gomes 1 \\ Gustavo Velásquez-Meléndez ${ }^{1}$
}

\section{Resumo}

Trata-se de um estudo transversal que avaliou indicadores de saúde cardiovascular em 863 indivíduos adultos residentes em comunidades rurais do Vale do Jequitinhonha, Minas Gerais, Brasil. Foram avaliados sete fatores, quatro comportamentais (dieta, atividade física, tabagismo e peso corporal) e três biológicos (colesterol total, glicemia em jejum e pressão arterial), classificados em níveis ideal, intermediário e ruim. Considerando os fatores comportamentais os homens tiveram melhor desempenho $(p=0,01)$, sendo observado um maior número de homens com 2 e 3 fatores em níveis ideais (48,5 e 25,8\%, respectivamente), em detrimento das mulheres $(44,2$ e 19,8\%, respectivamente) ( $p=0,01)$. Por outro lado, as mulheres apresentaram maior número de fatores biológicos em níveis ideais $(19,1 \%)$ quando comparadas com os homens $(7,9 \%)$ ( $p<$ 0,001). Somente três $(0,4 \%)$ participantes apresentaram os sete fatores na categoria ideal. A prevalência dos indicadores em nível ideal foi muito baixa, demonstrando a urgência de estratégias visando melhorar a saúde cardiovascular desta população.

Doenças Cardiovasculares; População Rural; Fatores de Risco; Promoção da Saúde 


\section{Introdução}

Na população mundial e também na brasileira as doenças crônicas não transmissíveis (DCNT) se tornaram atualmente um grande desafio para os sistemas de saúde. Perante o cenário mundial favorável ao aumento da expectativa de vida, à concentração proporcional de mortes em grupos etários acima de 70 anos, à diminuição da carga global de doença por malária e HIV/AIDS e à leve queda da mortalidade infantil abaixo de 5 anos de forma geral, as DCNT assumiram um papel central na atribuição da mortalidade e de sobrevida com incapacidades 1. Cabe ressaltar que as camadas pobres da população são as mais afetadas por este cenário 2 .

A quantificação do impacto das doenças crônicas na saúde em nível global tem sido realizada por meio de esforços acadêmicos da comunidade científica que colocaram em evidência diversos cenários da saúde global como o aumento do controle das doenças infecciosas e substancial progresso na redução de mortes prematuras por doenças cardiovasculares. Desta forma, as doenças crônicas e seus fatores de risco têm se mostrado como os maiores causadores de incapacidades na última década 1,3 . Por exemplo, a pressão arterial elevada, o consumo de álcool e a dieta inadequada representam os maiores fatores de risco para morte e doenças incapacitantes em todo o mundo 4,5 e também no Brasil 1.

Neste sentido, políticas públicas que contemplem ações de intervenções consideradas efetivas e de elevado custo/benefício por resultarem rapidamente em aumento da sobrevida e redução da incidência dessas doenças têm sido altamente desejáveis 6. No Brasil, o Plano de Ações Estratégicas para o Enfrentamento das Doenças Crônicas Não Transmissíveis no Brasil 2011-2022 tem-se pautado na redução da taxa de mortalidade prematura (< 70 anos) por DCNT, redução da prevalência de obesidade e do consumo nocivo de álcool, aumento da prevalência de atividade física no lazer, melhora do consumo alimentar com foco em frutas e hortaliças e baixo consumo de sódio, e redução da prevalência de tabagismo em adultos 7 .

Recentemente, foram desenvolvidas definições de saúde cardiovascular associadas a sobrevida sem incapacidades e a menor mortalidade por doença cardiovascular. Estes são baseados em 4 fatores comportamentais: não fumar, praticar atividade física regularmente, ter índice de massa corporal (IMC) $<25 \mathrm{~kg} / \mathrm{m}^{2}$ e uma dieta saudável; e a 3 fatores biológicos: colesterol $<200 \mathrm{mg} / \mathrm{dL}$, pressão arterial $<120 / 80 \mathrm{mmHg}$ e glicemia de jejum $<100 \mathrm{mg} / \mathrm{dL}^{8}$.
Nos últimos 10 anos, a população urbana brasileira apresentou importante redução do tabagismo, um pouco mais de $50 \% 7$. Apesar disso, os níveis de atividade física no lazer na população adulta são baixos (30\%); apenas 20,2\% consomem cinco ou mais porções diárias de frutas e hortaliças; $34,6 \%$ consomem carnes com elevado teor de gordura e $29,8 \%$ consomem refrigerantes 5 ou mais dias por semana; o que contribui para o aumento da prevalência de excesso de peso e obesidade, que atingem $48,5 \%$ e $15,8 \%$ dos adultos, respectivamente 9 . Cabe ressaltar, também, que as populações rurais apresentam maiores problemas de saúde quando comparadas à urbana, e os problemas cardiovasculares são o principal motivo do absenteísmo ou da não realização das atividades habituais 10 . Esta desvantagem entre o meio urbano/rural ainda piora, devido à menor oferta, menor complexidade dos serviços e pior qualidade da assistência de saúde na área rural $11,12,13$, reduzindo o acesso ao tratamento e às ações de promoção e prevenção à saúde. Ademais, pouca atenção é dada à saúde das populações rurais.

Dentro deste contexto, o objetivo deste trabalho foi avaliar a prevalência de fatores comportamentais e fatores biológicos em níveis ideais em uma população rural brasileira.

\section{Métodos}

\section{Desenho, local e população de estudo}

Trata-se de um estudo transversal conduzido no período entre 2008 e 2010, nas comunidades de Virgem das Graças, área rural do Município de Pontos dos Volantes; Caju e São Pedro do Jequitinhonha, áreas rurais do Município de Jequitinhonha; municípios situados na região do Vale do Jequitinhonha, Minas Gerais, Brasil.

As áreas rurais estudadas localizam-se em uma região semi-árida e de acesso restrito por estradas pavimentadas. $\mathrm{O}$ acesso aos serviços de saúde e de educação também é limitado. O comércio, a pecuária de corte, o garimpo e o cultivo de mandioca, milho, feijão, arroz e banana são as principais atividades econômicas da população. A população reside em casas construídas com tijolo de barro e telhas sem rede pública de água nem de esgoto 14 .

A partir de um censo foram registrados 937 adultos disponíveis para estudo, com idade igual ou superior a 18 anos, ambos os sexos, residentes nessas comunidades. Entretanto, foram excluídas as mulheres grávidas ( $\mathrm{n}=3$ ), as que não sabiam se estavam grávidas ( $n=6)$, e os participantes que não realizaram antropometria, coleta de 
sangue, ou não responderam as questões sobre dieta, tabagismo e atividade física $(n=65)$, resultando numa amostra final de 863.

\section{Coleta de dados}

Os dados foram coletados entre abril de 2008 a junho de 2011, por equipe composta por enfermeiros e nutricionistas, previamente treinados, que realizaram entrevista face-a-face. O questionário tinha perguntas sobre características sociodemográficas, hábitos alimentares, estilo de vida, e, ao final, os participantes foram submetidos a uma avaliação clínica que incluiu antropometria, aferição da pressão arterial e coleta de sangue para análise bioquímica. As medidas antropométricas foram aferidas três vezes segundo recomendações padronizadas 15, e a média utilizada. Também foram realizadas três medidas da pressão arterial seguindo-se recomendações internacionais 16. A coleta de aproximadamente $25 \mathrm{~mL}$ de sangue em jejum de 12 horas foi realizada para dosagem sérica da glicemia, triglicérides, colesterol total e fracionado.

\section{Avaliação da saúde cardiovascular}

Para avaliar os indicadores de saúde cardiovascular dos participantes, foi utilizado o escore de saúde cardiovascular, com adaptação da proposta original da American Heart Association 8. Trata-se de sete indicadores, sendo 4 fatores comportamentais: dieta, atividade física, peso corporal e tabagismo, e 3 fatores biológicos: glicemia de jejum, colesterol total e pressão arterial. Cada componente foi classificado em três categorias: ideal, intermediária e ruim, conforme os critérios descritos abaixo. A "saúde cardiovascular ideal" foi definida como a presença simultânea de todos os sete fatores em níveis ideais.

\section{- Fatores comportamentais}

\section{a) Dieta}

Consumo de salada crua, frutas, e verduras/legumes com as opções de resposta: todos os dias, 5-6 vezes por semana, 1-4 vezes por semana e nunca/quase nunca, foram questionados. Para avaliar a qualidade da dieta foram atribuídas pontuações ao consumo alimentar dos participantes de acordo com a Tabela 1. A soma dos pontos de cada resposta para cada indivíduo originou uma escala que pode variar de 0-6. Considerou-se como dieta ideal os indivíduos que apresentaram 6 pontos, dieta intermediária 5 pontos e dieta ruim igual ou menor que 4 pontos. A consistência interna desta escala foi analisada por meio do cál- culo do alfa de Cronbach 17, obtendo-se um valor de 0,62 , considerado aceitável.

\section{b) Atividade física}

A atividade física foi aferida em quatro domínios - domicílio, trabalho, lazer e deslocamento - por meio da versão longa do Questionário Internacional de Atividade Física (IPAQ) 18. Os minutos por semana de atividade física para cada indivíduo foram calculados, sendo consideradas apenas as atividades moderadas e vigorosas. Atividade física ideal foi classificada como $\geq 150$ minutos/ semana, intermediária 1-149 minutos/semana e ruim quando não realizava atividade física moderada ou vigorosa.

\section{c) Peso corporal}

O peso foi aferido utilizando balança eletrônica portátil (Marte, São Paulo, Brasil) calibrada e o indivíduo utilizava roupas leves, sem sapatos ou meias. A altura foi determinada com auxílio de antropômetro vertical (Alturexata, Belo Horizonte, Brasil) no $0,1 \mathrm{~cm}$ mais próximo, em posição ereta, pés juntos e cabeça no plano de Frankfurt 15. A partir dessas medidas foi calculado o IMC $=$ peso/ altura $^{2}$. O IMC ideal foi considerado quando menor que $25 \mathrm{~kg} / \mathrm{m}^{2}$, intermediário quando entre $25-29,9 \mathrm{~kg} / \mathrm{m}^{2}$, incluindo esses valores, e ruim quando $\geq 30 \mathrm{~kg} / \mathrm{m}^{2}$.

\section{d) Tabagismo}

Este comportamento foi classificado como ideal se a pessoa nunca fumou, intermediário na condição de ex-fumante e ruim quando fumante atual à data da coleta.

\section{- Fatores biológicos}

\section{a) Glicemia de jejum e colesterol total}

As concentrações bioquímicas foram determinadas por meio de método enzimático colorimétrico com auxílio do analisador COBAS MIRA PLUS (Roche Diagnostics, Suíça). O colesterol total ideal deveria estar menor que $200 \mathrm{mg} / \mathrm{dL}$, o intermediário entre $200-239 \mathrm{mg} / \mathrm{dL}$ e o ruim $\geq 240 \mathrm{mg} /$ $\mathrm{dL}$. A glicose menor que $100 \mathrm{mg} / \mathrm{dL}$ foi considerada ideal, entre $100-125 \mathrm{mg} / \mathrm{dL}$ intermediária e $\geq 126 \mathrm{mg} /$ dL ruim.

\section{b) Pressão arterial}

Os níveis pressóricos foram classificados como ideais quando menores que $120 / 80 \mathrm{mmHg}$, intermediários se pressão arterial sistólica (PAS) 
Pontuação utilizada para avaliar o consumo alimentar da população estudada.

\begin{tabular}{lcccc}
\hline Alimento & & \multicolumn{2}{c}{ Frequência semanal } \\
& Todo dia & $\mathbf{5 - 6}$ x/semana & $\mathbf{1 - 4}$ x/semana & Quase nunca/Nunca \\
\hline Salada crua & 2 & 2 & 1 & 0 \\
Frutas & 2 & 2 & 1 & 0 \\
Verduras/Legumes & 2 & 2 & 1 & 0 \\
\hline
\end{tabular}

entre $120-139 \mathrm{mmHg}$ ou pressão arterial diastólica (PAD) entre $80-89 \mathrm{mmHg}$, e ruim quando PAS $\geq 140 \mathrm{mmHg}$ ou PAS $\geq 90 \mathrm{mmHg}$.

\section{Índice de fatores comportamentais e biológicos}

Os fatores comportamentais e fatores biológicos descritos acima foram agrupados, também conforme proposto pela American Heart Association, originando dois índices: índice de fatores comportamentais e índice de fatores biológicos 8 .

$\mathrm{O}$ índice de fatores comportamentais, que está associado à sobrevida sem incapacidade 8 , foi dimensionado considerando somente os 4 fatores, assim os participantes podem ter entre 0 e 4 fatores comportamentais em nível ideal.

O índice de fatores biológicos, que se associa a menor mortalidade por doença cardiovascular 8 contempla os 3 fatores biológicos e um comportamental, o hábito de fumar. Desta forma, os participantes podem ter entre 0 e 4 fatores biológicos em níveis ideais.

Ressalta-se que o tabagismo é um componente de ambos os índices, pois ele está associado tanto à sobrevida sem doença quanto à menor mortalidade 8 .

\section{Análise estatística}

Os dados foram analisados usando o programa estatístico Stata, versão 12.0 (Stata Corp., College Station, Estados Unidos). A caracterização da população segundo variáveis sociodemográficas e sexo foi realizada por meio de cálculo das frequências absolutas e relativas. A análise dos índices propostos foi realizada segundo o sexo e as diferenças estatísticas foram avaliadas por meio do teste qui-quadrado de Pearson $(\mathrm{p}<0,05)$.

\section{Questões éticas}

Este estudo foi aprovado pelo Comitê de Ética em Pesquisa da Universidade Federal de Minas
Gerais, conforme Resolução no 196/96 do Conselho Nacional de Saúde, segundo parecer ETIC $604 / 07$. Todos os indivíduos que fizeram parte do estudo foram informados sobre os objetivos da pesquisa e sobre seus direitos como participantes e, voluntariamente, assinaram o Termo de Consentimento Livre e Esclarecido.

\section{Resultados}

Observou-se uma distribuição relativamente homogênea em relação ao sexo e às faixas etárias. A escolaridade da população é baixa, cerca de $20 \%$ eram analfabetos e aproximadamente $45 \%$ possuíam de 1-4 anos de estudo. A maioria é casada (65,6\%) (dados não mostrados).

A análise dos fatores individuais do escore de saúde cardiovascular mostrou que a maioria dos indivíduos foi classificada na categoria saúde ideal, exceto para hipertensão arterial e dieta, para os quais a prevalência da classificação de saúde ruim foi de 30,9 e $86,4 \%$, respectivamente (Tabela 2).

Observa-se que a distribuição das categorias de saúde cardiovascular por sexo não foi similar para a maioria dos componentes do escore. Maiores prevalências de escore de saúde cardiovascular na categoria ruim no grupo de mulheres foram observados para os fatores: atividade física $(20,1 \%)$, peso corporal $(15,3 \%)$ e colesterol $(23,5 \%)$. No grupo dos homens as prevalências de escore na categoria ruim foram altas para tabagismo $(30,4 \%)$, dieta $(89,5 \%)$ e pressão arterial $(31,9 \%)$, essas diferenças foram consideradas estatisticamente significativas $(\mathrm{p}<0,05)$.

Analisando o índice de fatores comportamentais (Figura 1) observou-se baixa prevalência (1,0\%) de indivíduos com os quatro fatores comportamentais em níveis ideais, independente do sexo. Ademais, um maior número de homens com 2 e 3 fatores em níveis ideais (48,5 e 25,8\%, respectivamente) foi observado, em detrimento das mulheres (44,2 e 19,8\%, respectivamente) 
Distribuição dos participantes segundo número de fatores comportamentais ideais (0-4) para a população total, homens e mulheres. Virgem das Graças, Caju, São Pedro do Jequitinhonha, Minas Gerais, Brasil, 2008-2010.

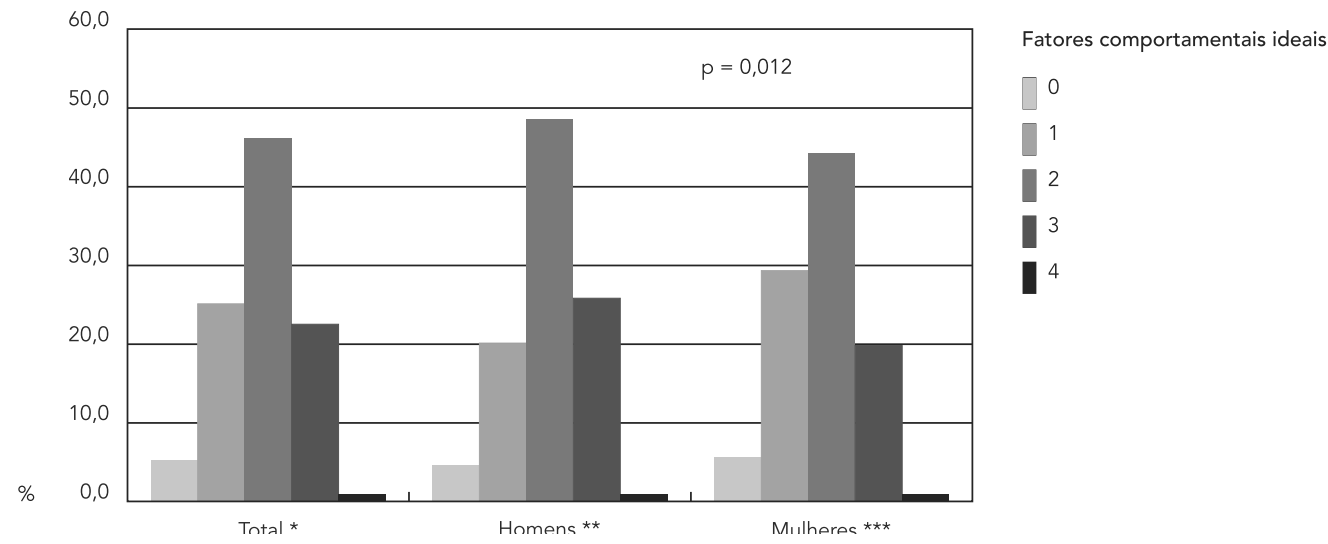

$$
\begin{aligned}
& { }^{\star} \mathrm{n}=909 ; \\
& \star \star n=418 ; \\
& \star \star \star n=491 .
\end{aligned}
$$

Fatores comportamentais: não fumar; IMC $<25 \mathrm{~kg} / \mathrm{m}^{2}$; fisicamente ativo; consumo de frutas, verduras e legumes de 5-7 dias por semana.

( $p=0,01)$. Em contrapartida, observou-se no índice de fatores biológicos que as mulheres apresentam maior número dos quatro fatores biológicos em níveis ideais $(19,1 \%)$, quando comparadas com os homens $(7,9 \%)(p<0,001)$ (Figura 2).

Os homens apresentaram maior número de fatores em níveis ideais, em relação às mulheres $(\mathrm{p}=0,03)$. Somente três participantes $(0,4 \%)$ da população estudada, todos eles homens, apresentaram os sete fatores componentes do escore de saúde cardiovascular na categoria ideal. Seis indivíduos $(0,7 \%)$ não apresentaram fatores em níveis ideais. A maioria da população $(72,7 \%)$ apresentou quatro ou menos fatores em nível ideal (Figura 3). Nenhum indivíduo apresentou todos os fatores componentes do escore em nível ruim (dados não mostrados).

\section{Discussão}

Os resultados da avaliação dos indicadores de saúde cardiovascular obtidos neste estudo indicaram baixos níveis de adequação à dieta saudável, altas frequências de hipertensão e alta prevalência de fumantes. Um recente relatório sobre a carga global de doenças mostrou que os fatores: dieta inadequada, hipertensão, IMC elevado, tabagismo, glicemia de jejum elevada e inatividade, nessa ordem de importância, estão dentre os que contribuem em maior magnitude para a causa de mortes e de anos de vida perdidos ajustados por incapacidade na população brasileira 1. Dessa forma a população estudada potencialmente apresentaria uma diminuição da sobrevida sem incapacidades e elevado risco de mortalidade por doenças cardiovasculares conforme indicado pela baixa frequência de indivíduos da população que apresentaram os sete fatores em nível ideal (Figura 3).

A maioria da população foi classificada como ideal na avaliação isolada dos fatores, exceto para os fatores dieta e pressão arterial, cujas prevalências em níveis ideais foram 3,9\% e 31,5\%, respectivamente (Tabela 2 ). $\mathrm{O}$ achado referente ao consumo inadequado de frutas e hortaliças é esperado tanto na população urbana quanto na rural. A última Pesquisa de Orçamentos Familiares (POF) mostrou que somente $2,8 \%$ das calorias ingeridas diariamente eram provenientes destes alimentos 19 , sendo o esperado de $6 \%$ a $7 \%$. Esse mesmo inquérito encontrou, ainda, menor consumo destes alimentos em homens do que em mulheres e no meio rural em comparação ao urbano 19,20. Dados recentes do sistema de vigilância 21 também mostram baixa aderência 
Figura 2

Distribuição dos participantes segundo número de fatores biológicos ideais (0-4) para a população total, homens e mulheres. Virgem das Graças, Caju, São Pedro do Jequitinhonha, Minas Gerais, Brasil, 2008-2010.

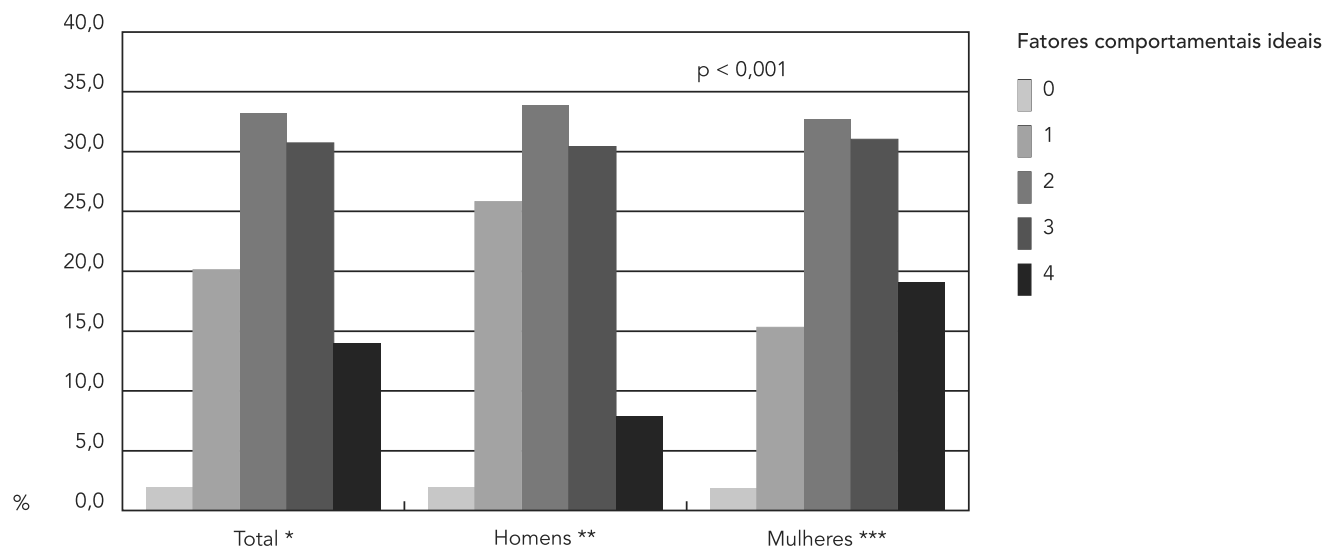

${ }^{\star} \mathrm{n}=872$

** $\mathrm{n}=395$;

$\star \star \star n=477$

Fatores biológicos: não fumar; colesterol total < 200mg/dL; pressão arterial sistólica $<120 \mathrm{mmHg}$ e pressão arterial diastólica $<80 \mathrm{mmHg}$ (sem tratamento); e glicose em jejum < 100mg/dL (sem tratamento).

Figura 3

Distribuição dos participantes segundo número de fatores de saúde cardiovascular ideais (0-7) para a população total, homens e mulheres. Virgem das Graças, Caju, São Pedro do Jequitinhonha, Minas Gerais, Brasil, 2008-2010.

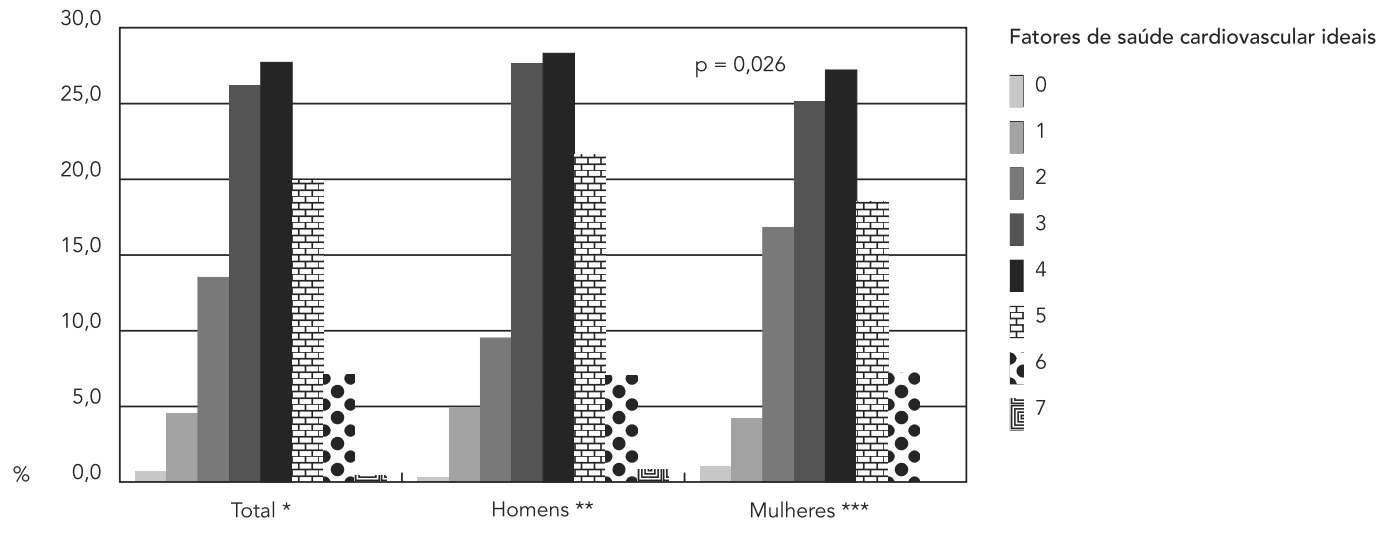

$$
\begin{aligned}
& { }^{*} \mathrm{n}=863 ; \\
& \star \star n=388 ; \\
& \star \star \star n=475 .
\end{aligned}
$$

Fatores de saúde cardiovascular ideal: não fumar; IMC $<25 \mathrm{~kg} / \mathrm{m}^{2}$; fisicamente ativo; consumo de frutas, verduras e legumes de 5-7 dias por semana; colesterol total $<200 \mathrm{mg} / \mathrm{dL}$; pressão arterial sistólica $<120 \mathrm{mmHg}$ e pressão arterial diastólica $<80 \mathrm{mmHg}$ (sem tratamento); e glicose em jejum < 100mg/dL (sem tratamento). 
Tabela 2

Classificação dos indicadores de saúde cardiovascular em nível ideal, intermediário e ruim, segundo sexo. Virgem das Graças, Caju, São Pedro do Jequitinhonha, Minas Gerais, Brasil, 2008-2010.

\begin{tabular}{|c|c|c|c|c|}
\hline Fatores & $\begin{array}{c}\text { Feminino } \\
\text { n (\%) }\end{array}$ & $\begin{array}{c}\text { Masculino } \\
\text { n (\%) }\end{array}$ & Valor $p$ & $\begin{array}{l}\text { Total } \\
\text { n (\%) }\end{array}$ \\
\hline \multicolumn{5}{|l|}{ Tabagismo $(\mathrm{n}=919)$} \\
\hline Ideal & $362(73,3)$ & $165(38,8)$ & $<0,001$ & $527(57,4)$ \\
\hline Intermediário & $72(14,5)$ & $131(30,8)$ & & $203(22,1)$ \\
\hline Ruim & $60(12,2)$ & $129(30,4)$ & & $189(20,5)$ \\
\hline Escore da dieta $(n=920)$ & & & 0,017 & \\
\hline Ideal & $20(4,1)$ & $16(3,7)$ & & $36(3,9)$ \\
\hline Intermediário & $61(12,3)$ & $29(6,8)$ & & $90(9,7)$ \\
\hline Ruim & $413(83,6)$ & $381(89,5)$ & & $794(86,4)$ \\
\hline Atividade física $(n=914)$ & & & $<0,001$ & \\
\hline Ideal & $230(46,6)$ & $346(82,2)$ & & $576(63,1)$ \\
\hline Intermediário & $164(33,3)$ & $24(5,7)$ & & $188(20,5)$ \\
\hline Ruim & $99(20,1)$ & $51(12,1)$ & & $150(16,4)$ \\
\hline Peso corporal $(n=923)$ & & & $<0,001$ & \\
\hline Ideal & $280(56,8)$ & $320(74,4)$ & & $600(65,1)$ \\
\hline Intermediário & $138(27,9)$ & $89(20,7)$ & & $227(24,5)$ \\
\hline Ruim & $75(15,3)$ & $21(4,9)$ & & $96(10,4)$ \\
\hline Glicose $(n=882)$ & & & 0,464 & \\
\hline Ideal & $436(91,2)$ & $377(93,3)$ & & $813(92,2)$ \\
\hline Intermediário & $29(6,1)$ & $20(4,9)$ & & $49(5,5)$ \\
\hline Ruim & $13(2,7)$ & $7(1,8)$ & & $20(2,3)$ \\
\hline Colesterol $(n=881)$ & & & 0,004 & \\
\hline Ideal & $240(50,2)$ & $229(56,8)$ & & $469(53,2)$ \\
\hline Intermediário & $126(26,3)$ & $115(28,6)$ & & $241(27,4)$ \\
\hline Ruim & $112(23,5)$ & $59(14,6)$ & & $171(19,4)$ \\
\hline Pressão arterial ( $n=928)$ & & & 0,004 & \\
\hline Ideal & $178(35,9)$ & $114(26,3)$ & & $292(31,5)$ \\
\hline Intermediário & $168(33,9)$ & $181(41,8)$ & & $349(37,6)$ \\
\hline Ruim & $149(30,2)$ & $138(31,9)$ & & $287(30,9)$ \\
\hline
\end{tabular}

a uma dieta saudável, sendo que somente $27,2 \%$ e $17,6 \%$ de mulheres e homens das capitais brasileiras, respectivamente, relataram consumo de cinco ou mais porções por dia, em 5 ou mais dias da semana. Estudos similares ao presente estudo, que avaliam fatores relacionados a promoção da saúde cardiovascular em outras populações, confirmam a falta de adequação a dietas saudáveis 1,22,23,24.

Uma potencial explicação para o baixo consumo de frutas e hortaliças no meio rural está relacionada ao acesso a esses alimentos, visto este ser influenciado por períodos de safra e entre safra o que afeta principalmente o consumo de frutas 25 e pelo clima, onde a seca predomina sobre a época de chuvas, o que dificulta o cultivo da terra 26 e justifica a dificuldade de plantio e produção de verduras e legumes. Destaca-se, ainda, a baixa renda 27 e a ausência de implementação das políticas públicas voltadas a promoção de alimentação saudável 26. Para concretização dessas políticas deve considerar os aspectos biológicos, socioculturais dos indivíduos e da coletividade, bem como o uso sustentável do meio ambiente, desenvolvendo habilidades pessoais em alimentação e nutrição saudável. Além disso, devem transcender as unidades de saúde com inserção nos demais equipamentos sociais 28 .

Outro indicador de saúde cardiovascular avaliado foi o peso corporal. A maior prevalência de excesso de peso nas mulheres é consistente com estudos prévios em nível nacional. Pesquisa recente sobre peso corporal da população brasileira encontrou uma prevalência de excesso de 
peso em $41,3 \%$ e $50,2 \%$ respectivamente em homens e mulheres do sudeste rural 29. Evidências sugerem que essa prevalência diferencial entre os sexos, além dos fatores biológicos, pode ser explicada pelo maior desequilíbrio energético positivo nas mulheres 19,29. Neste estudo, além da dieta inadequada observada na maior parte da população, verificou-se maior proporção de inatividade física nas mulheres, uma vez que suas atividades são predominantemente restritas ao ambiente domiciliar. Estudo nesta mesma população mostrou que grande percentual dos moradores concentra suas atividades físicas nos domínios trabalho (82,9\%), domicílio $(63,5 \%)$ e deslocamento (32\%), sendo a atividade física no trabalho a que mais contribuiu com a atividade de intensidade moderada ou vigorosa 30 , o que justifica o comportamento ativo dos homens e menor proporção de excesso de peso.

Em relação ao tabagismo mais da metade dos homens avaliados fumam ou já fumaram. Atualmente, cerca de $17 \%$ da população brasileira fuma, sendo que esta prevalência pode alcançar níveis maiores dependendo do sexo (21,6\% para homens) e de características sociodemográficas (25,7\% entre os menos escolarizados) 31 . Políticas públicas de combate ao tabagismo têm tido bastante sucesso, com redução de $50 \%$ na prevalência nos últimos 20 anos 32 . Portanto, apesar da queda alcançada, o tabagismo continua importante problema de saúde pública e certamente na população estudada, pois o consumo de cigarros se concentra em populações de baixa renda, de menor escolaridade e residentes em área rural 31.

Quanto aos fatores biológicos a hipertensão arterial e hipercolesterolemia foram os fatores mais prevalentes entre os homens e as mulheres, respectivamente. No Brasil, em 2011, 22,7\% ( $25,4 \%$ mulheres e $19,5 \%$ homens) e $5,6 \%$ (5,2\% homens e $6 \%$ mulheres) dos indivíduos maiores de 18 anos apresentavam diagnóstico de hipertensão arterial e diabetes, respectivamente 9 . Em relação à dislipidemia, 14,1\% dos homens e $19,3 \%$ das mulheres com 18 anos ou mais relataram diagnóstico médico de dislipidemia em 2009 33. A maioria dos estudos se baseia em dados autorreferidos podendo subestimar a prevalência da doença. Entretanto, as prevalências encontradas nesta população podem ser mais realistas uma vez que se apoiam em aferições diretas, justificando maiores frequências de hipertensão e de hipercolesterolemia.

Considerando a análise agregada dos indicadores, resultados de estudos internacionais que aplicaram o escore proposto pela American Heart Association também mostraram prevalências muito baixas, com diferenças entres os se- xos 22,23,24,34,35,36. Diferentemente dos resultados encontrados em populações americanas 23,35, em nosso estudo o cenário foi desfavorável às mulheres, marcado pelo pior desempenho no índice de fatores comportamentais, porém os homens apresentaram pior desempenho no índice de fatores biológicos. Em geral no Brasil as mulheres relatam maior morbidade e utilizam com mais frequência os serviços de saúde 11 . Por outro lado, estudos que avaliaram somente os comportamentos de saúde de forma isolada observaram maior exposição a fatores de risco comportamentais para doenças cardiovasculares em homens, enquanto fatores protetores foram mais frequentes nas mulheres 37,38 .

As inequidades em saúde em populações rurais, tais como dificuldade de acesso a cuidados oportunos 24 e à informação sobre hábitos de vida saudáveis 12,26,28 em relação às comunidades urbanas podem potencialmente explicar os resultados encontrados. No Brasil as necessidades de serviços de saúde em comunidades rurais são supridas pela equipe de Estratégia Saúde da Família (ESF). Em todas as áreas de estudo pode ser encontrada uma unidade básica de saúde (UBS), presença de agente comunitário de saúde e técnico de enfermagem diariamente e visitas programadas semanais pela enfermeira e quinzenalmente pelo médico 39; e em São Pedro do Jequitinhonha, unidade com maior infraestrutura, o funcionamento é diário, e atendimento do médico e do enfermeiro de duas a três vezes por semana 40. Entretanto, estudo sobre o acesso a serviços de saúde na região evidenciou aspectos organizacionais que afetam a oferta de serviços, bem como dificuldades de acesso decorrentes das características topográficas das áreas geográficas onde estão localizadas as comunidades 39 . Dessa forma, as altas prevalências de indicadores em níveis intermediário e ruim podem ser atribuídas ao baixo acesso a serviços de saúde e má qualidade dos serviços, o que pode influenciar negativamente a prevenção e a continuidade de tratamentos direcionados a estas condições crônicas.

O cenário encontrado nesta população rural pode contribuir fortemente para níveis altos de anos de vida perdidos ajustados por incapacidade e com isso a mortes prematuras, perda de qualidade de vida, alto grau de limitação das pessoas em suas atividades de trabalho e de lazer, além de ocasionar impactos econômicos negativos para as famílias, as comunidades e a sociedade em geral 7. Como a população rural é aproximadamente $15 \%$ da população brasileira, os resultados deste estudo podem potencialmente dar uma dimensão das doenças crônicas na população rural brasileira. 
O presente estudo apresenta algumas limitações que devem ser listadas. A primeira está relacionada à definição dos fatores avaliados, pois foram realizadas adaptações dos indicadores decorrentes da ausência de informações, tais como: tempo que o indivíduo parou de fumar, consumo de medicamentos e diagnóstico de diabetes. Da mesma forma, a aferição da dieta foi baseada apenas na referência ao consumo de frutas e hortaliças e não a outros marcadores de consumo alimentar como sódio, peixes e grãos como originalmente proposto. Este marcador de qualidade de dieta também foi utilizado anteriormente para avaliar a saúde cardiovascular em população americana 35 e o consumo de 5-7 vezes por semana de cada um dos alimentos investigados (frutas, saladas cruas e verduras/ legumes) sugere um padrão alimentar composto por alimentos minimamente processados, contribuindo para baixa densidade energética e alta densidade de nutrientes (fibras, vitaminas e minerais) da dieta 41 , constituindo-se um indicador protetor de saúde cardiovascular. Outra adaptação foi que neste estudo a atividade física foi contabilizada levando em consideração todos os domínios e não só no lazer. Sabe-se que os novos paradigmas das relações entre atividades físicas e saúde mostram que é primordial a avaliação das práticas em outros domínios como no trabalho e no ambiente doméstico 42 .

A segunda limitação esta relacionada à validade externa, apesar de se tratar de um estudo com uma amostra relativamente grande da população rural esta pode não ser representativa. Em contrapartida, pesquisas com amostras representativas de padrão de consumo das populações rurais confirmam o sentido dos nossos achados 1,20. Ademais, acreditamos que o cenário encontrado pode ser o de várias populações rurais brasileiras que apresentam características semelhantes à da população estudada.

Em resumo, este estudo mostrou que uma alta proporção da população rural apresentou baixas prevalências de indicadores de saúde cardiovascular em níveis ideais, com diferenças entre os sexos, para o qual os homens apresentaram maior potencial de risco de mortalidade e as mulheres, maior potencial de risco de desenvolver doenças ao longo da vida. De uma forma geral, o cenário registrado neste estudo é de impacto negativo sobre os anos de vida perdidos ajustados por incapacidades e a carga global de doenças, com predisposição ao aumento de anos de vida potencialmente perdidos e desenvolvimento de incapacidades ao longo da vida.

Estes resultados podem auxiliar no reconhecimento de que a promoção da saúde cardiovascular continua sendo precária nas áreas rurais e na implementação das metas do Plano de Ações Estratégicas para o Enfrentamento das Doenças Crônicas Não Transmissíveis no Brasil 20112022, proposto recentemente pelo Ministério da Saúde. Assim, as políticas públicas de saúde deveriam ser prioritárias para essas populações, que se beneficiariam de ações de promoção e prevenção à saúde.

\section{Resumen}

Se trata de un estudio transversal que evaluó los indicadores de la salud cardiovascular en 863 adultos que viven en las comunidades rurales del Valle de Jequitinhonha, Minas Gerais, Brasil. Se describen siete factores, cuatro conductuales (dieta, actividad física, el tabaquismo y el peso corporal) y tres biológicos (colesterol total, glucemia y presión arterial), clasificados en los siguientes niveles: óptimo, intermedio y malo. Teniendo en cuenta los factores conductuales, los hombres obtuvieron mejores resultados ( $p=0,01)$, y se observó un mayor número de hombres con factores de 2 y 3 en niveles óptimos (48,5 y 25,8\%, respectivamente), en detrimento de las mujeres $(44,2$ y 19,8\%, respectivamente) ( $p=0,01)$. Por otro lado, las mujeres tenían un mayor número de factores biológicos en niveles óptimos (19,1\%), en comparación con los hombres (7,9\%) ( $p<$ 0,001). Sólo tres $(0,4 \%)$ participantes tenían los siete factores categorizados en un nivel ideal. La prevalencia de estos indicadores en un nivel óptimo es muy baja, lo que demuestra la urgencia de estrategias para mejorar la salud cardiovascular de esta población.

Enfermedades Cardiovasculares; Población Rural; Factores de Riesgo; Promoción de la Salud 


\section{Colaboradores}

M. S. Felisbino-Mendes participou do planejamento da pesquisa, coleta de dados, análise estatística, interpretação dos dados, redação do artigo, revisão crítica relevante do conteúdo intelectual e aprovação da versão final do manuscrito. A. K. Jansen participou da interpretação dos dados, redação do artigo, revisão crítica relevante do conteúdo intelectual e aprovação da versão final do manuscrito. C. S. Gomes participou da análise estatística, interpretação dos dados, redação do artigo, revisão crítica relevante do conteúdo intelectual e aprovação da versão final do manuscrito. G. Velásquez-Meléndez foi responsável pela concepção e projeto, supervisão, redação do artigo, revisão crítica do conteúdo intelectual e aprovação da versão final do manuscrito.

\section{Referências}

1. Institute for Health Metrics and Evaluation. Global burden of disease profile: Brazil, 2013. http:// www.healthmetricsandevaluation.org/gbd/coun try-profiles (accessado em 30/Abr/2013).

2. Schmidt I, Duncan BB, Silva GA, Menezes AM, Monteiro CA, Barreto SM, et al. Chronic non-com municable diseases in Brazil: burden and current challenges. Lancet 2011; 377:1949-61.

3. World Health Organization. Global status report on noncommunicable diseases 2010. Geneva: World Health Organization; 2011.

4. Fuster V, Kelly B. Summary of the Institute of Medicine Report. Global Heart 2011; 6:133-42.

5. World Health Organization. World health statistics 2012. Geneva: World Health Organization; 2012.

6. World Health Organization/World Heart Federation/World Stroke Organization. Global atlas on cardiovascular disease prevention and control. Geneva: World Health Organization; 2011.

7. Coordenação Geral de Doenças e Agravos Não Transmissíveis, Departamento de Análise de Situação de Saúde, Secretaria de Vigilância em Saúde, Ministério da Saúde. Plano de ações estratégicas para o enfrentamento das doenças crônicas não transmissíveis (DCNT) no Brasil 2011-2022. Brasília: Ministério da Saúde; 2011. (Série B. Textos Básicos de Saúde).

\section{Agradecimentos}

Agradecemos à FAPEMIG e ao CNPq pelo apoio financeiro para a realização deste estudo. Agradecemos também à professora Andréa Gazzinelli Corrêa de Oliveira pela coordenação do trabalho de campo deste estudo.
8. Lloyd-Jones DM, Hong Y, Labarthe D, Mozaffarian D, Appel LJ, Van Horn L, et al. Defining and setting national goals for cardiovascular health promotion and disease reduction: The American Heart Association's Strategic Impact Goal through 2020 and beyond. Circulation 2010; 121:586-613.

9. Secretaria de Vigilância em Saúde, Ministério da Saúde. Vigitel Brasil 2011. Vigilância de fatores de risco e proteção para doenças crônicas por inquérito telefônico. Brasília: Ministério da Saúde, 2012. (Série G. Estatística e Informação em Saúde).

10. Kassouf AL. Acesso aos serviços de saúde nas áreas urbana e rural do Brasil. Revista de Economia e Sociologia Rural 2005; 43:29-44.

11. Pinheiro RS, Viacava F, Travassos C, Brito AS. Gênero, morbidade, acesso e utilização de serviços de saúde no Brasil. Ciênc Saúde Coletiva 2002; 7:687-707.

12. Carneiro FF, Tambellini AT, Silva JA, Haddad JPA, Búrigo AC, de Sá WR, et al. Saúde de famílias do movimento dos trabalhadores sem terra e de bóias-frias, Brasil, 2005. Rev Saúde Pública 2008; 42:727-63.

13. O'Donnell W. Access to health care in developing countries: breaking down demand side barriers. Cad Saúde Pública 2007; 23:2820-34. 
14. Gazzinelli A, Hightower A, LoVerde PT, Haddad JP, Pereira WR, Bethony J, et al. The spatial distribution of Schistosoma mansoni infection before and after chemotherapy in the Jequitinhonha Valley in Brazil. Mem Inst Oswaldo Cruz 2006; 101 Suppl. 1:S63-71.

15. Lohman TG, Roche AF, Martorell R. Anthropometric standardization reference manual. Champaign: Human Kinetics Books; 1988.

16. Chobanian AV, Bakris GL, Black HR, Cushman WC, Green LA, Izzo JR. JL, et al. The Seventh Report of the Joint National Committee (JNC) on prevention, detection, evaluation, and treatment of high blood pressure. Hypertension 2003; 42:1206-52.

17. Maroco J, Garcia-Marques T. Qual a fiabilidade do alfa de Cronbach? Questões antigas e soluções modernas? Laboratório de Psicologia 2006; 4:65-90.

18. Matsudo S, Araujo TVM, Matsudo V, Andrade D, Andrade E, Oliveira LC, et al. Questionário internacional de atividade física (IPAQ): estudo de validade e reprodutibilidade no Brasil. Rev Bras Ativ Fís Saúde 2001; 6:5-18.

19. Levy RB, Claro RM, Mondini L, Sichieri R, Monteiro CA. Regional and socioeconomic distribution of household food availability in Brazil, in 20082009. Rev Saúde Pública 2012; 46:6-15.

20. Instituto Brasileiro de Geografia e Estatística. Pesquisa de Orçamentos Familiares - POF 2008-2009. Análise do consumo alimentar pessoal no Brasil. Rio de Janeiro: Instituto Brasileiro de Geografia e Estatística; 2011.

21. Departamento de Vigilância de Doenças e Agravos não Transmissíveis e Promoção de Saúde, Secretaria de Vigilância em Saúde, Ministério da Saúde. Vigitel Brasil 2012. Vigilância de fatores de risco e proteção para doenças crônicas por inquérito telefônico. http://portalsaude.saude.gov.br/portal saude/arquivos/pdf/2013/Ago/27/coletiva_vigi tel_270813.pdf (acessado em 20/Out/2013).

22. Graciani A, León-Muñoz LM, Guallar-Castillón P, Rodríguez-Artalejo F, Banegas JR. Cardiovascular health in a Southern Mediterranean European country. Circ Cardiovasc Qual Outcomes 2013; 6:90-8.

23. Folsom AR, Yatsuya H, Nettleton JA, Lutsey LP, Cushman M, Rosamond WD. Community prevalence of ideal cardiovascular health, by the AHA definition, and relationship with cardiovascular disease incidence. J Am Coll Cardiol 2011; 57:1690-6.

24. Kim JI, Sillah A, Boucher JL, Sidebottom AC, Knickelbine T. Prevalence of the American Heart Association's "Ideal Cardiovascular Health" metrics in a rural, cross-sectional, community-based study: The Heart of New Ulm Project. J Am Heart Assoc 2013; 2:e000058.

25. Carvalho EO, Rocha EF. Consumo alimentar de população adulta residente em área rural da cidade de Ibatiba (ES, Brasil). Ciênc Saúde Coletiva 2011; 16:179-85.
26. Coordenação Geral da Política de Alimentação e Nutrição, Departamento de Atenção Básica, Secretaria de Atenção à Saúde, Ministério da Saúde. Diagnóstico de saúde e nutrição da população do campo: levantamento de dados. Brasília: Ministério da Saúde; 2004.

27. Claro RM, Monteiro CA. Family income, food prices, and household purchases of fruits and vegetables in Brazil. Rev Saúde Pública 2010; 44:1-6.

28. Departamento de Atenção Básica, Secretaria de Atenção à Saúde, Ministério da Saúde. Política Nacional de Alimentação e Nutrição. Brasília: Ministério da Saúde; 2012. (Série B. Textos Básicos de Saúde).

29. Instituto Brasileiro de Geografia e Estatística. Pesquisa de Orçamentos Familiares - POF 2008-2009. Antropometria e estado nutricional de crianças, adolescentes e adultos no Brasil. Rio de Janeiro: Instituto Brasileiro de Geografia e Estatística; 2010.

30. Bicalho PG, Hallal PC, Gazzinelli A, Knuth AG, Velásquez-Meléndez G. Atividade física e fatores associados em adultos de área rural em Minas Gerais, Brasil. Rev Saúde Pública 2010; 44:884-93.

31. Facina T. Pesquisa especial de tabagismo (PETab) relatório Brasil. Rev Bras Cancerol 2011; 57:429-30.

32. Levy D, Almeida LM, Szklo A. The Brazil Sim Smoke Policy Simulation Model: the effect of strong tobacco control policies on smoking prevalence and smokig-attributable deaths in a middle income nation. PLoS Med 2012; 9:e1001336.

33. Secretaria de Gestão Estratégica e Participativa, Secretaria de Vigilância em Saúde, Ministério da Saúde. Vigitel Brasil 2009. Vigilância de fatores de risco e proteção para doenças crônicas por inquérito telefônico. Brasília: Ministério da Saúde; 2010.

34. Wu H-Y, Sun ZH, Cao DP, Wu LX, Zeng Q. Cardiovascular health status in Chinese adults in urban areas: analysis of the Chinese Health Examination Database 2010. Int J Cardiol 2012; 168:760-4.

35. Bambs CE, Kip KE, Dinga A, Mulukutla SR, Aiyer NA, Reis SE. Low prevalence of "ideal cardiovascular health" in a community-based population: the Heart strategies concentrating on risk evaluation (Heart SCORE) study. Circulation 2011; 123:850-7.

36. Shay CM, Ning H, Allen NB, Carnethon MR, Chiuve SE, Greenlund KJ, et al. Status of cardiovascular health in US adults: prevalence estimates from the National Health and Nutrition Examination Surveys (NHANES) 2003-2008. Circulation 2012; 125:45-56.

37. Iser BPM, Yokota RTC, Sá NNB, Moura L, Malta DC. Prevalência de fatores de risco e proteção para doenças crônicas nas capitais do Brasil - principais resultados do Vigitel 2010. Ciênc Saúde Coletiva 2012; 17:2343-56.

38. Malta DC, Moura EC, Morais Neto OL. Gender and schooling inequalities in risk and protective factors for chronic diseases among Brazilian adults, through telephone survey. Rev Bras Epidemiol 2011; 14:125-35. 
39. Vieira EWR. Acesso e utilização dos serviços de saúde de Atenção primária em população rural do município de Jequitinhonha, Minas Gerais [Dissertação de Mestrado]. Belo Horizonte: Escola de Enfermagem, Universidade Federal de Minas Gerais; 2010.

40. Reis DC. Acesso da população ao diagnóstico e tratamento da esquistossomose em área endêmica do município de Jequitinhonha, Minas Gerais [Tese de Doutorado]. Belo Horizonte: Escola de Enfermagem, Universidade Federal de Minas Gerais; 2009 .
41. Monteiro CA. Nutrition and health. The issue is not food, nor nutrients, so much as processing. Public Health Nutr 2009; 12:729-31.

42. Malta DC, Moura EC, Castro AM, Cruz DKA, Morais Neto OL, Monteiro CA. Padrão de atividade física em adultos brasileiros: resultados de um inquérito por entrevistas telefônicas, 2006. Epidemiol Serv Saúde 2009; 18:7-16.

Recebido em 21/Mai/2013

Versão final resubmetido em 27/Nov/2013

Aprovado em 03/Dez/2013 